\title{
LncRNA HITT Overexpression in Gastric Cancer Promotes Cell Apoptosis by Suppressing the Maturation of miR-602
}

\section{Yun Chen}

Department of Gastroenterology $₫$ The first Affiliated Hospital of Gannan Medical College, Ganzhou City, Jiangxi Province, 341000, PR. China

\section{Canhui Ouyang}

Department of Gastroenterology, The first Affiliated Hospital of Gannan Medical College, Ganzhou City, Jiangxi Province, 341000, PR. China

\section{Lingyun Liao}

Department of Gastroenterology, The first Affiliated Hospital of Gannan Medical College, Ganzhou City, Jiangxi Province, 341000, PR. China

\section{Yun Zhou}

Department of Gastroenterology, The first Affiliated Hospital of Gannan Medical College, Ganzhou City, Jiangxi Province, 341000, PR. China

\section{Fan Meng}

Department of Gastroenterology, The first Affiliated Hospital of Gannan Medical College, Ganzhou City, Jiangxi Province, 341000, PR. China

\section{Yao Liu}

Department of Gastroenterology, The first Affiliated Hospital of Gannan Medical College, Ganzhou City, Jiangxi Province, 341000, PR. China

\section{Jing Ye ( $\square$ jingyejiangxi@163.com )}

Office of Academic Affairs of Jiangxi University of Traditional Chinese Medicine, Nanchang City, Jiangxi Province, 330004, PR. China

\section{Research Article}

Keywords: HITT, gastric cancer, miR-602, apoptosis

Posted Date: December 21st, 2020

DOl: https://doi.org/10.21203/rs.3.rs-128931/v1

License: (c) (i) This work is licensed under a Creative Commons Attribution 4.0 International License. Read Full License 


\section{Abstract}

Background: HITT inhibits colon cancer. This study explored its role in gastric cancer (GC).

Methods: HITT, mature miR-602 and premature miR-602 expression in paired GC and normal tissues (62 patients) was studied by RT-qPCR. RNA pull-down assay was performed to analyze the direct interaction between HITT and mature miR-602. The subcellular location of HITT was analyzed by nuclear fractionation assay. The role of HITT in regulating miR-602 maturation was analyzed by overexpression assay. Cell apoptosis was analyzed by flow cytometry.

Result: Our assays illustrated that HITT was highly expressed in GC and mature miR-602 was lowly expressed in GC. No alteration in premature miR-602 in GC was observed. HITT was located to both nucleus and cytoplasm, and it can directly interact with miR-602. HITT overexpression in GC cells increased the expression of mature miR-602 but not premature miR-602. HITT overexpression increased GC cell apoptosis and suppressed the role of miR-602 in inhibiting GC cell apoptosis.

Conclusion: Therefore, HITT may promote GC cell apoptosis by suppressing the maturation of miR-602.

\section{Introduction}

As a major burden of global health, gastric cancer (GC) affects about 1 million people worldwide each year (1). At present, patients with early stage GC in many cases can be cured by surgical resection (2). However, in most countries except Japan, most GC patients are diagnosed with inoperable conditions, or developed recurrent tumors after resection with curative intent $(3,4)$. Once tumor metastasis to distant ends has occurred, surgical resection will be inappropriate (5). Although chemotherapy or radio therapy can be applied to treat GC, full recovery is rare and prognosis is generally poor (6). Therefore, the field of GC treatment is awaiting more effective therapeutic approaches.

With the advantages of less adverse effects and higher accuracy, molecular targeted therapies are emerging novel approaches for the treatment of cancers including $\mathrm{GC}(7,8)$. For instance, regulating the expression of HER2 and miR-374a-5p, which play critical roles in GC, has shown potentials in the treatment of advanced GC $(9,10)$. However, more therapeutic targets are still needed to achieve better treatment outcomes (11). MiRNAs and IncRNAs are non-coding RNAs that have no coding capacity but regulate protein synthesis to participate in most, if not all types of cancers including GC $(12,13)$.

Therefore, IncRNAs and miRNAs are promising targets for GC treatment. A recent study showed that HITT could inhibit colon cancer (14). Our preliminary microarray analysis showed the altered expression of HITT in GC. In addition, HITT was closely correlated with miR-602, which is an important player in cancer biology (15). We then analyzed the interaction between HITT and miR-602 in GC.

\section{Material And Methods}

Tissue specimens 
HITT is a novel IncRNA and all public datasets lack its expression data. To investigate the expression pattern of HITT in GC, 62 paired GC and non-tumor tissue specimens were obtained from the first Affiliated Hospital of Gannan Medical College (Ethics Committee of this hospital approved this study). The specimens were stored at $-80^{\circ} \mathrm{C}$ prior to following assays. The tumor types and stages were analyzed, diagnosed and confirmed by 3 experienced pathologists. The 62 cases included 30 cases of gastric adenocarcinoma (GA) and 32 cases of gastric carcinoma (GC). Based on AJCC staging system, the 62 cases included 28 cases at stage I or II, and 34 cases at stage III or IV. All these patients signed informed consent. Based on medical record, none of these patients received therapy prior to the collection of specimens.

\section{GC cells}

To match the patients included in this study, this study included SNU-1 (carcinoma) and AGS (adenocarcinoma) two GC cell lines, which were purchased from ATCC (USA). These cells were cultivated in 1640 medium (10\% FBS). Regular mycoplasma test and STR profiling were perfromed (Mykoalert detection kit).

\section{Cell transfections}

SNU-1 and AGS cells were overexpressed with HITT and miR-602 through transfections mediated by Neon Transfection System (Thermo Fisher Scientific), in which miR-602 mimic or pcDNA3.1- HITT expression vector (Invitrogen) was used. In all cases, two controls, including negative control (empty vector- or NC miRNA-transfection) and control (untransfected cells) were included. The overexpression of HITT and miR-602 was confirmed every $24 \mathrm{~h}$ until the end of all assays that included overexpression experiments.

\section{RNA samples and RT-qPCRs}

Following RNA extractions using RNAzol reagent (Sigma-Aldrich), genomic removal was performed using DNase I (Sangon). Genomic DNA digestion in all cases was performed until the ratio of OD260/280 reached about 2.0, which was used as the indicator or pure RNA samples. Urea-PAGE gels (4.5\%) were used to separate RNA samples, following by ethidium bromide staining and visulization under UV lights.

To detect the expression of HITT and premature miR-218, RNA samples were first used to prepare cDNA samples. With cDNA samples as template and 18S rRNA as internal control, qPCRs were carried out to measure the expression levels of HITT across samples. The expression of mature miR-218 was analyzed using All-in-One ${ }^{\mathrm{TM}}$ miRNA qRT-PCR Detection Kit (Genecopoeia). The method of $2^{-\triangle \Delta C T}$ was used for the normalization of Ct values.

\section{Nuclear fractionation assay}

SNU-1 and AGS cells were used to prepare nuclear and cytosol samples with BioVision Nuclear/Cytosol Fractionation Kit (\# K266). Both samples were subjected to the preparation of RNA samples through the 
methods mentioned above. The same amount of RNA samples were first used to prepare cDNA samples, which were used to perform semi-quantitative PCR amplifications. Different PCR cycles were used to avoid over-amplification. In this experiment, amplification of GAPDH was performed as a positive control.

\section{RNA pull-down assay}

Biotinylated premature miR-602 (Bio-premature miR-602) and NC (Bio-NC) were transfected into SNU-1 and AGS cells. At 48h post-transfection, cell lysis was prepared and was subjected to separation using Streptaviden magnetic beads (Invitrogen). After that, HITT expression was analyzed by RT-qPCR.

\section{Cell apoptosis analysis}

SNU-1 and AGS cells were harvested at the time point of 48h post-transfection. These cells were cultivated in non-serum medium for $48 \mathrm{~h}$ at $37^{\circ} \mathrm{C}$. After that, cells were washed with ice-cold PBS twice and then staining with FITC Annexin-V and PI (Abcam) was carried out in dark for $15 \mathrm{~min}$. Apoptotic cells were analyzed by flow cytometry.

\section{Statistical analysis}

Heatmaps plotted using Heml 1.0 software were used to present the differential gene expression in paired tissue samples. Data collected from different cell transfection groups (more than 3 ) was compared by ANOVA Tukey's test. $P<0.05$ was statistically significant.

\section{Results}

\section{HITT and mature miR-602, but not premature miR-602 were altered in GC}

HITT, premature miR-602 and mature miR-602 in the 62 pairs of tissues were analyzed. Data was inputted into Heml 1.0 software to plot Heatmaps. Our data illustrated that HITT was lowly expressed in GC tissues (Fig.1A). In contrast, mature miR-602 was lowly expressed in GC tissues (Fig.1B). To our surprise, expression levels of premature miR-602 were not obviously different between paired tissues (Fig.1C).

\section{HITT was located to both nucleus and cytoplasm}

Nuclear fractionation assay was performed to determine the subcellular location of HITT. Our data illustrated that, similar to GAPDH, HITT was localized to both nucleus and cytoplasm (Fig.2). Therefore, HITT may traffic from nucleus to cytoplasm.

\section{HITT was inversely correlated with mature miR-602 across GC samples and suppressed the maturation of miR-602 in GC cells}

Pearson's correlation coefficient analysis showed that, across GC tissues, HITT was inversely and closely correlated with the expression of mature miR-602 (Fig.3A). In contrast, HITT and mature miR-602 were not closely correlated across GC tissues (Fig.3B). SNU-1 and AGS cells were overexpressed with HITT and 
miR-602, and the overexpression was confirmed every 24h until 96h (Fig.3C, p<0.05). Our data illustrated that HITT overexpression significantly decreased the expression levels of mature miR-602 in both cell lines (Fig.3D, $p$ <0.05). However, no significant alterations in expression levels of mature miR-602 were observed (Fig.3E, p < 0.05). Moreover, no significant alteration in expression of HITT was observed after miR-602 overexpression (Fig.3F).

\section{HITT overexpression increased GC cell apoptosis through miR-602}

The roles of HITT and miR-602 in regulating the apoptosis of SNU-1 and AGS cells were analyzed by cell apoptosis assay. Our data illustrated that, HITT overexpression increased GC cell apoptosis, while miR602 overexpression inhibited cell apoptosis. Moreover, HITT overexpression suppressed the role of miR602 in inhibiting GC cell apoptosis (Fig.4, p<0.05).

\section{HITT directly interacted with premature miR-602}

RNA pull-down assay was performed to analyze the direct interaction between HITT. Compared to bio-NC group, bio- premature miR-602 group exhibited significantly higher levels of HITT, suggesting the direct interaction between HITT and premature miR-602 (Fig.5, p<0.05).

\section{Discussion}

The expression of HITT in GC and its interaction with miR-602 was explored in this study. We found that HITT was highly expressed in GC. In addition, HITT may suppress the maturation of miR-602 to induce the apoptosis of GC.

HITT is a recently characterized IncRNA with tumor suppressive role in colon cancer (14). It was observed that HITT was lowly expressed in colon cancer and the downregulation of HIFF is required for the expression of HIF-1a induced by hypoxia, thereby regulating angiogenesis and tumor growth (14). Based on our knowledge, the role of HITT in other cancers is unknown. In this study we showed that HITT was downregulated in GC, and the overexpression of HITT increased the apoptosis of GC cells. Therefore, HITT is likely a tumor suppressor in GC and overexpression of HITT may serve as a potential target for the treatment of GC.

The oncogenic role of miR-502 has been investigated in several types of cancers (16). For instance, miR602 is overexpressed in liver cancer and suppresses the expression of tumor suppressor RASSF1A to promote cancer development (16). In addition, miR-602 is also overexpressed in esophageal squamous cell carcinoma and forms a negative feedback loop with FOXK2 to regulate cancer progression (17). In this study we observed the upregulation of miR-602 in GC and its inhibitory effects on GC cell apoptosis, suggesting the oncogenic role of miR-602 in GC.

Interestingly, no significant alteration in the expression of premature miR-602 was observed in GC patients, suggesting that the maturation, but not transcription of miR-602 was altered in GC. We showed that HITT could suppress the maturation of miR-602 in GC cells. HITT was found to be located in both 
nucleus and cytoplasm. In addition, it can directly interact with premature miR-602. It has been well established that the movement of premature miRNAs from nucleus to cytoplasm is required for the maturation of miRNAs (18). We therefore speculated that HITT could suppress the movement of premature miR-602 to reduce the production of mature miR-602.

\section{Conclusion}

In conclusion, HITT is downregulated in GC and it may suppress the maturation of miR-602 to induce the apoptosis of GC.

\section{Declarations}

\section{Ethical Approval and Consent to participate}

Informed consent was obtained from all individual participants included in the study. All producers were approved by the first Affiliated Hospital of Gannan Medical College Ethics Committee. Procedures operated in this research were completed in keeping with the standards set out in the Announcement of Helsinki and laboratory guidelines of research in China.

\section{Consent to publish}

Not applicable.

\section{Availability of supporting data}

The data that support the findings of this study are available on request from the corresponding author.

The data are not publicly available due to their containing information that could compromise the privacy of research participants.

\section{Competing interests}

All other authors have no conflicts of interest.

We declare that we do not have any commercial or associative interest that represents a conflict of interest in connection with the work submitted.

\section{Funding}

Not Applicable.

\section{Authors' contributions}

YC, JY: study concepts, literature research, clinical studies, data analysis, experimental studies, manuscript writing and review; CHOY: study design, literature research, experimental studies and 
manuscript editing; LYL: definition of intellectual content, clinical studies, data acquisition and statistical analysis; YZ: data acquisition, manuscript preparation and data analysis; FM, YL: data acquisition and statistical analysis.

All authors have read and approve the submission of the manuscript.

\section{Acknowledgements}

Not Applicable.

\section{References}

1. Thrift AP, El-Serag HB. Burden of Gastric Cancer. Clinical gastroenterology and hepatology: the official clinical practice journal of the American Gastroenterological Association. 2020;18(3):534-42.

2. Yang K, Hu JK. Gastric cancer treatment: similarity and difference between China and Korea. Translational gastroenterology and hepatology. 2017;2:36.

3. Van Cutsem E, Sagaert X, Topal B, Haustermans K, Prenen H. Gastric cancer. Lancet. 2016;388(10060):2654-64.

4. Japanese gastric cancer treatment guidelines 2014 (ver. 4). Gastric cancer: official journal of the International Gastric Cancer Association and the Japanese Gastric Cancer Association. 2017;20(1):1-19.

5. Smyth EC, Moehler M. Late-line treatment in metastatic gastric cancer: today and tomorrow. Therapeutic advances in medical oncology. 2019;11:1758835919867522.

6. Ilson DH. Advances in the treatment of gastric cancer: 2019. Current opinion in gastroenterology. 2019;35(6):551-4.

7. Pellino A, Riello E, Nappo F, Brignola S, Murgioni S, Djaballah SA, et al. Targeted therapies in metastatic gastric cancer: Current knowledge and future perspectives. World journal of gastroenterology. 2019;25(38):5773-88.

8. Patel TH, Cecchini M. Targeted Therapies in Advanced Gastric Cancer. Current treatment options in oncology. 2020;21(9):70.

9. Meric-Bernstam F, Johnson AM, Dumbrava EEl, Raghav K, Balaji K, Bhatt M, et al. Advances in HER2Targeted Therapy: Novel Agents and Opportunities Beyond Breast and Gastric Cancer. Clinical cancer research: an official journal of the American Association for Cancer Research. 2019;25(7):2033-41.

10. Ji R, Zhang X, Gu H, Ma J, Wen X, Zhou J, et al. miR-374a-5p: A New Target for Diagnosis and Drug Resistance Therapy in Gastric Cancer. Molecular therapy Nucleic acids. 2019;18:320-31.

11. Figueiredo C, Garcia-Gonzalez MA, Machado JC. Molecular pathogenesis of gastric cancer. Helicobacter. 2013;18 Suppl 1:28-33.

12. Peng WX, Koirala P, Mo YY. LncRNA-mediated regulation of cell signaling in cancer. Oncogene. 2017;36(41):5661-7. 
13. Hao NB, He YF, Li XQ, Wang K, Wang RL. The role of miRNA and IncRNA in gastric cancer. Oncotarget. 2017;8(46):81572-82.

14. Wang X, Li L, Zhao K, Lin Q, Li H, Xue X, et al. A novel LncRNA HITT forms a regulatory loop with HIF$1 a$ to modulate angiogenesis and tumor growth. Cell death and differentiation. 2020;27(4):1431-46.

15. Zhou C, Huang Y, Chen Y, Xie Y, Wen H, Tan W, et al. miR-602 Mediates the RASSF1A/JNK Pathway, Thereby Promoting Postoperative Recurrence in Nude Mice with Liver Cancer. OncoTargets and therapy. 2020;13:6767-76.

16. Yang L, Ma Z, Wang D, Zhao W, Chen L, Wang G. MicroRNA-602 regulating tumor suppressive gene RASSF1A is overexpressed in hepatitis B virus-infected liver and hepatocellular carcinoma. Cancer biology \& therapy. 2010;9(10):803-8.

17. Liu M, Yu J, Wang D, Niu Y, Chen S, Gao P, et al. Epigenetically Upregulated MicroRNA-602 Is Involved in a Negative Feedback Loop with FOXK2 in Esophageal Squamous Cell Carcinoma. Molecular therapy: the journal of the American Society of Gene Therapy. 2019;27(10):1796-809.

18. Murchison EP, Hannon GJ. miRNAs on the move: miRNA biogenesis and the RNAi machinery. Current opinion in cell biology. 2004;16(3):223-9.

\section{Figures}

A

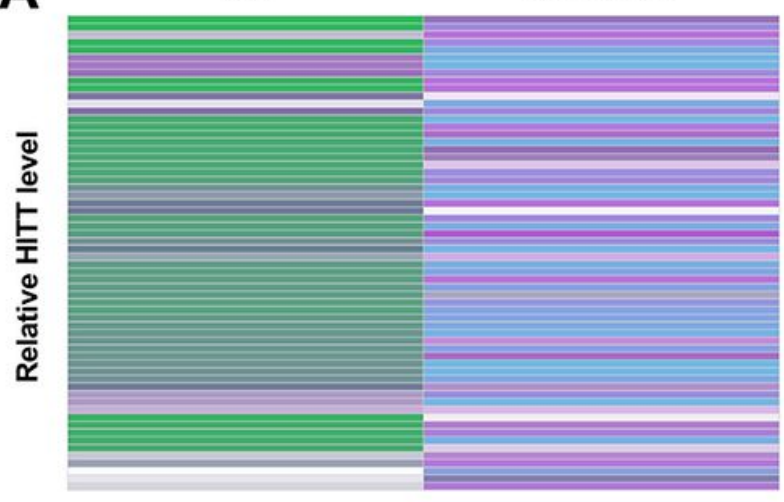

C

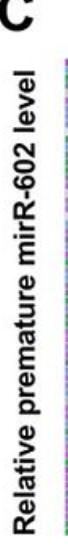

GC

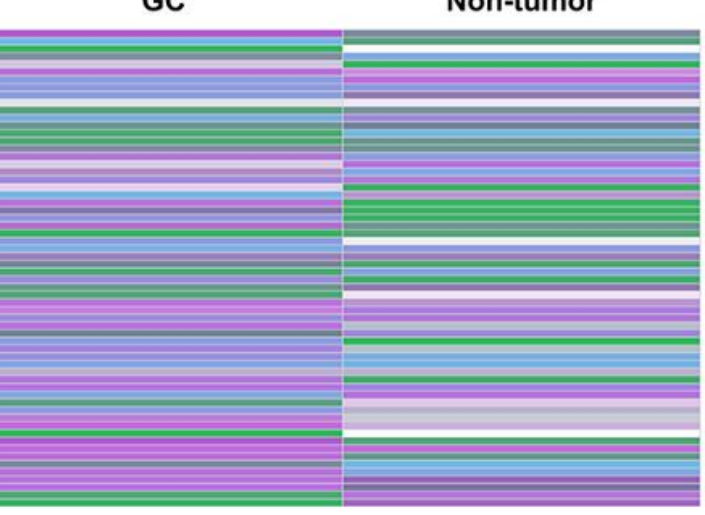

B

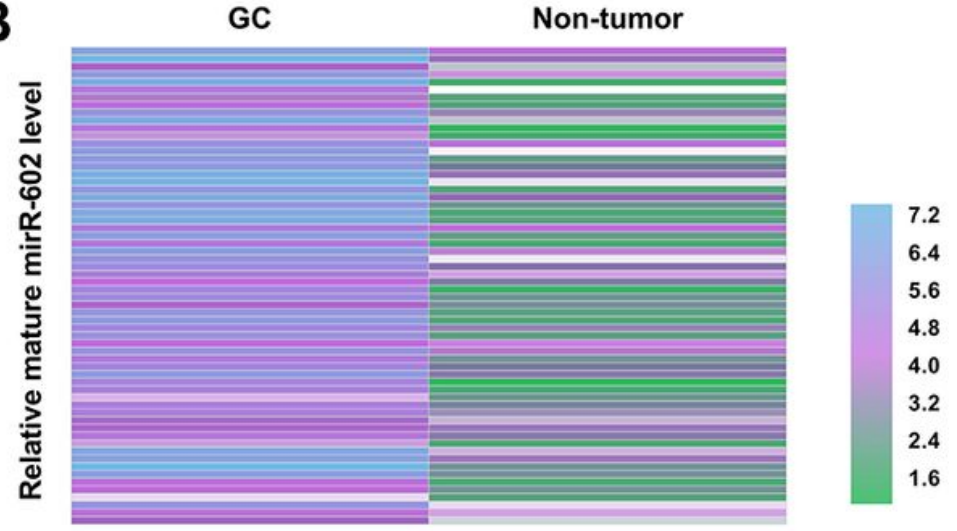

\section{Figure 1}


Analysis of the expression of HITT, premature miR-602 and mature miR-602 expression in GC HITT (A), mature miR-602 (B) and premature miR-602 (C) in the 62 pairs of tissues were analyzed. Data was inputted into Heml 1.0 software to plot Heatmaps.

\section{SNU-1}

\section{AGS}
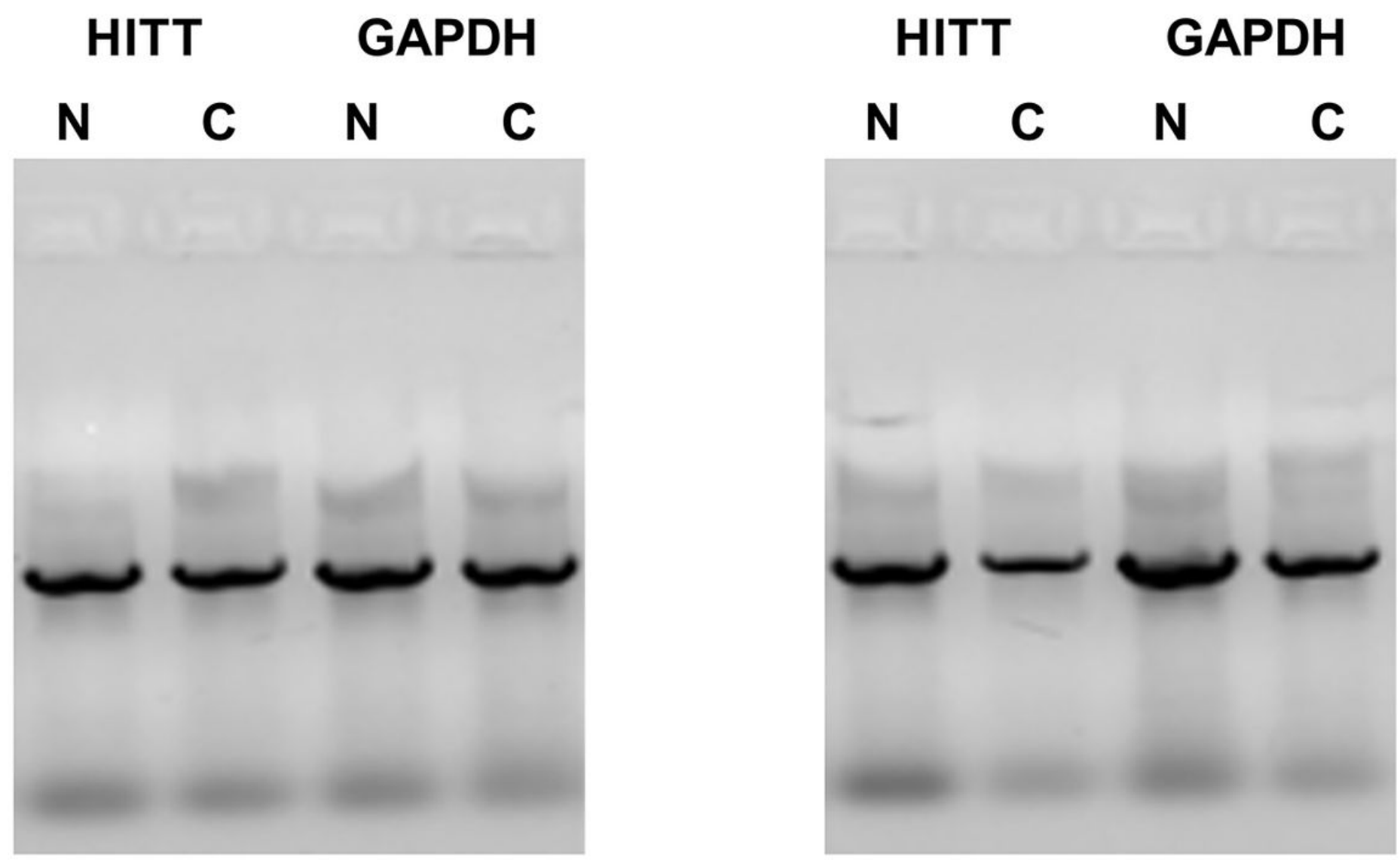

Figure 2

HITT was located to both nucleus and cytoplasm Nuclear fractionation assay was performed to determine the subcellular location of HITT. PCR products were subjected to $1 \%$ agarose gel electrophoresis, followed by EB staining. N, nucleus; C, cytoplasm. 

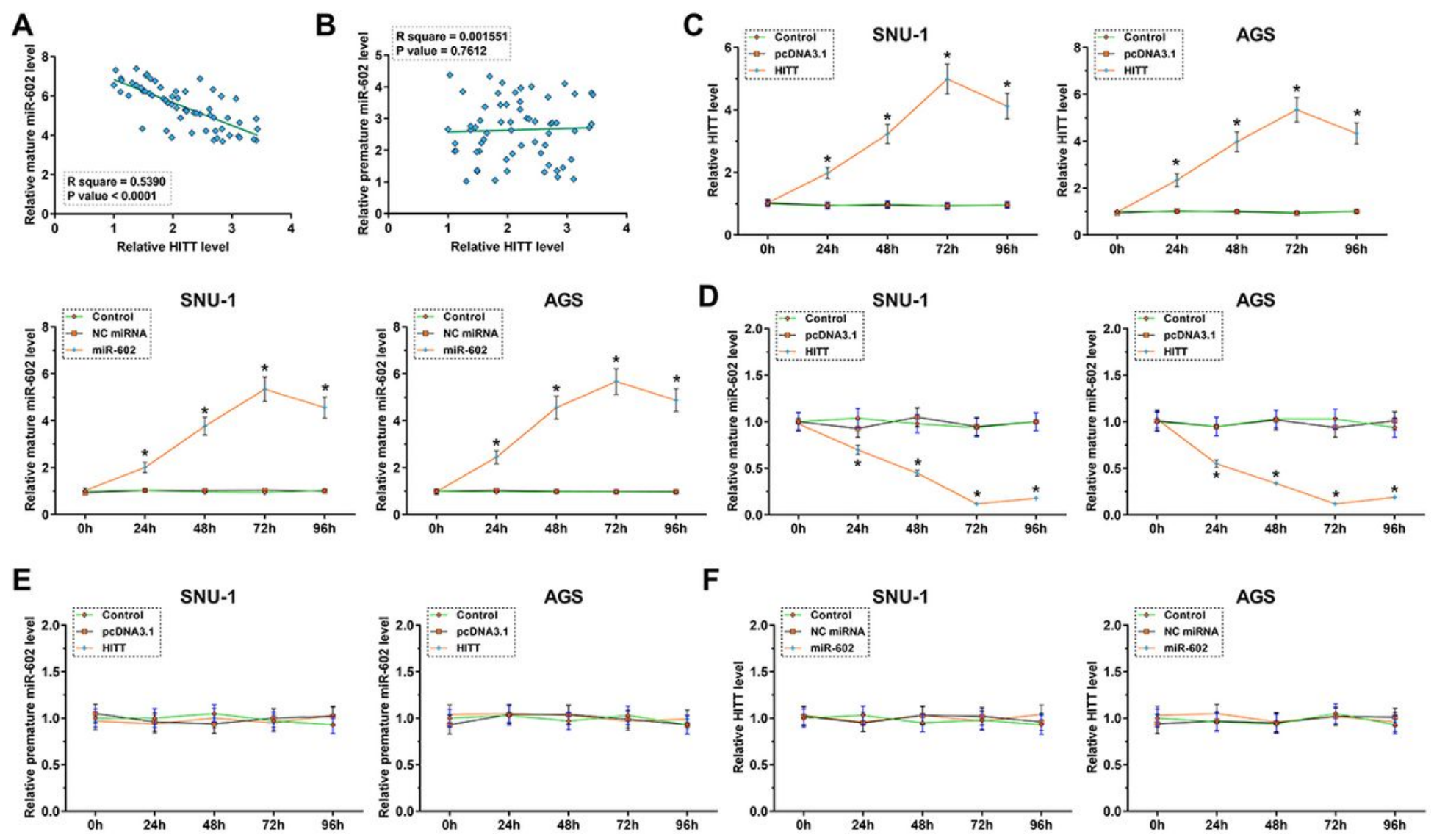

Figure 3

HITT was inversely correlated with mature miR-602 across GC samples and suppressed the maturation of miR-602 in GC cells Pearson's correlation coefficient analysis was performed to analyze the correlations between HITT and mature miR-602 (A) or premature miR-602 (B) across GC tissues. SNU-1 and AGS cells were overexpressed with HITT and miR-602, and the overexpression was confirmed every $24 \mathrm{~h}$ until $96 \mathrm{~h}$ (C). The alterations in mature miR-602 (D) and premature miR-602 (E) expression after HITT overexpression were analyzed by RT-qPCR. In addition, the alterations in expression levels of HITT after mature miR-602 overexpression were also analyzed by RT-qPCR $(F) .{ }^{*}, p<0.05$. 

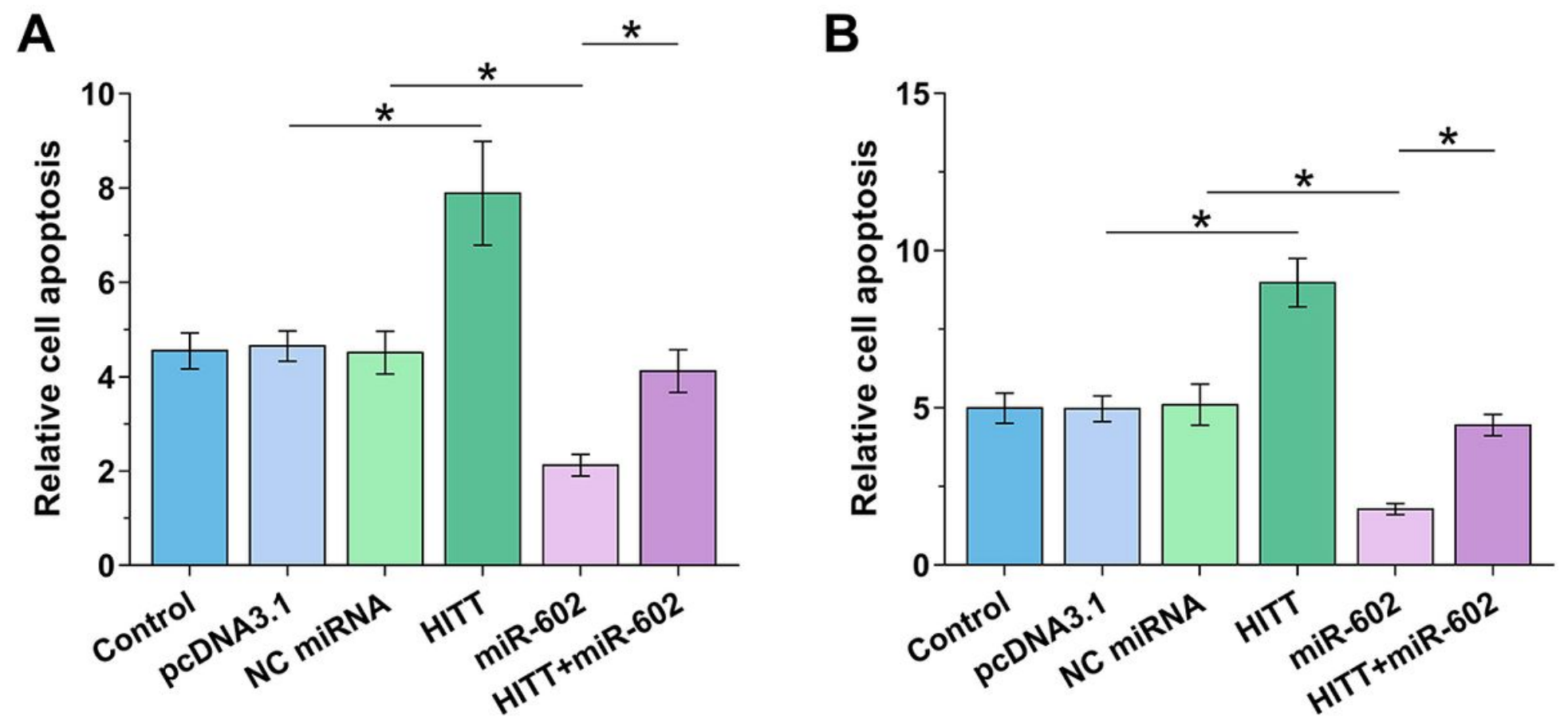

Figure 4

HITT overexpression increased GC cell apoptosis through miR-602 The roles of HITT and miR-602 in regulating the apoptosis of SNU-1 and AGS cells were analyzed by cell apoptosis assay. ${ }^{*}, p<0.05$.

SNU-1

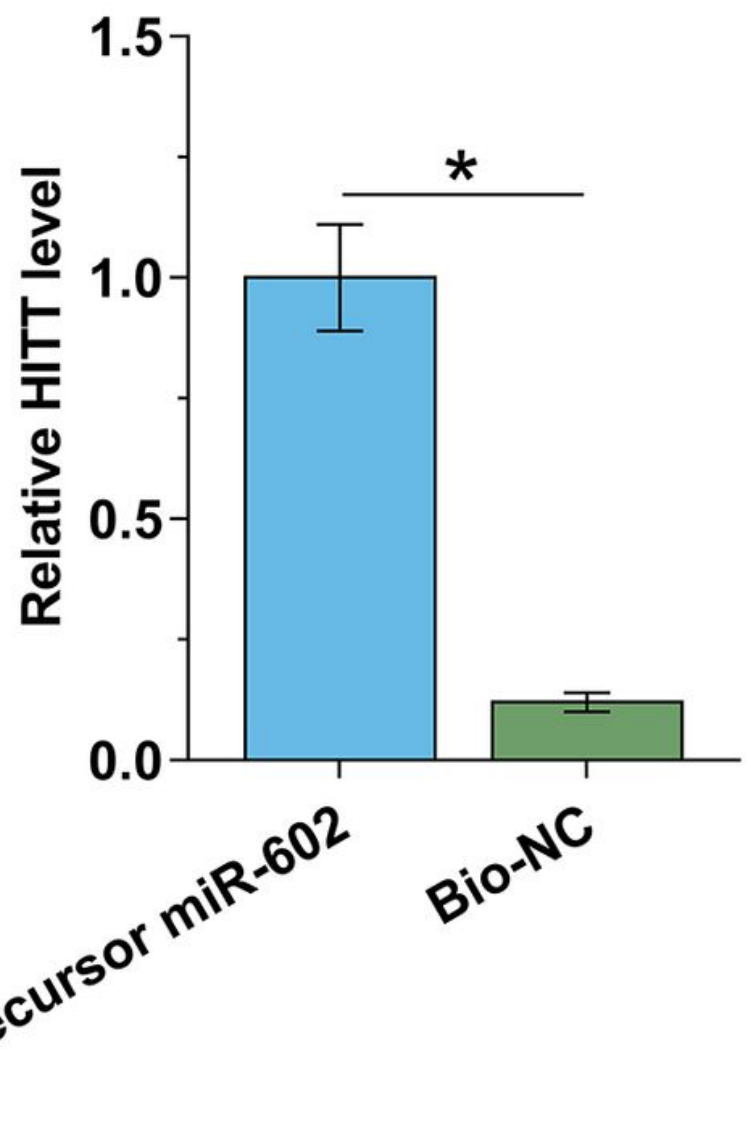

AGS

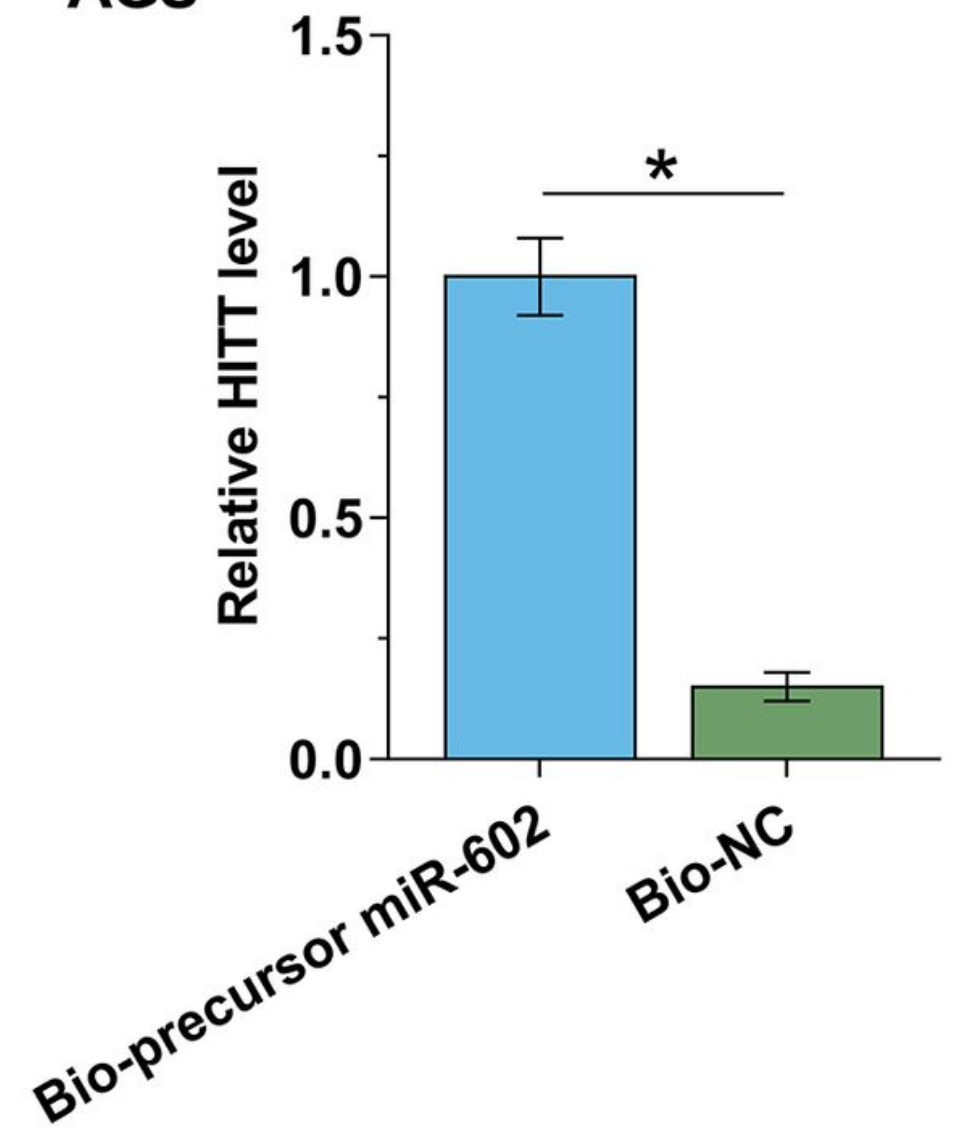

Figure 5 
HITT directly interacted with premature miR-602 RNA pull-down assay was performed to analyze the direct interaction between HITT. The RNA pull-down assay was performed following the methods described above. ${ }^{*}, \mathrm{p}<0.05$. 